\title{
O Programa Nacional de Saúde Reprodutiva de Cabo Verde: alcances, limites e desafios
}

\author{
The National Reproductive Health Programme in Cape Verde: \\ scope, limits and challenges
}

Wilza Vieira Villela ${ }^{1}$

Redy Wilson Lima ${ }^{2}$

Cláudia Fernandes de Brito ${ }^{3}$

${ }^{1}$ Departamento de Medicina Preventiva, Universidade Federal de São Paulo. R. Botucatu 740, Vila Clementino. 04023-062 São Paulo SP Brasil. wilsa. vieira@terra.com.br

${ }^{2}$ Centro Interdisciplinar de Ciências Sociais, Universidade Nova de Lisboa. Lisboa Portugal.

${ }^{3}$ Pesquisadora

Independente. São Paulo SP Brasil.
Abstract Cape Verde is an archipelago on the African coast, with 538,535 inhabitants living on nine islands. Since it gained independence in 1975, the country has experienced rapid economic and social growth, with significant gains in education as well as a reduction in maternal and infant mortality deaths. In 2001, following the guidelines of the International Conference on Population and Development (ICPD, Cairo, 1994), the National Reproductive Health Program (PNSR) was launched aiming to provide sexual and reproductive health (SRH) services to adolescents, young people and adult men, in partnership with the education sector and youth centers. However, the continuance of unplanned pregnancies, illegal abortions and HIV infections has indicated that there are gaps in the program's implementation. Studies conducted in the country point to socio-cultural aspects as well as aspects covering organizations and the services on offer, as some of the reasons for the identified problems. This paper aims to reflect on the scope and limits of the PNSR in Cape Verde with consideration being given to the challenges of implementing health policies that affect cultural practices related to gender and sexuality. Consideration is also given to the specifics of Cape Verde's demographics, economic and cultural aspects as well as the importance of the SRH services to its development.

Key words Public Policy, Sexual and Reproductive Health, Adolescents and Youth, Cape Verde
Resumo Cabo Verde é um arquipélago do continente africano, com cerca de 538.535 habitantes. Desde sua independencia, em 1975, o país tem experimentado um rápido crescimento econômico e social, com ganhos significativos em escolarização, redução dos óbitos maternos e da mortalidade infantil. Em 2001, seguindo as diretrizes da Conferencia Internacional de População e Desenvolvimento (CIPD, Cairo, 1994), foi lançado o Programa Nacional de Saúde Reprodutiva, PNSR, visando incluir ações de saúde sexual e reprodutiva, SSR, para adolescentes, jovens e homens adultos, em parceria com o setor de educação e centros de juventude. Entretanto, a persistência da gravidez não planejada, do aborto clandestino e da infecção pelo HIV indicam lacunas na sua implementação. Estudos realizados no país apontam razões socioculturais e no âmbito da organização e oferta de serviços para os problemas identificados. Este trabalho tem como finalidade refletir sobre os alcance e limites do PNSR de Cabo Verde, considerando os desafios de implementação de políticas de saúde que afetam práticas culturais relativas ao gênero $e$ a sexualidade; as especificidades demográficas, socioeconômicas e culturais de Cabo Verde e ainda a importancia da SSR para o desenvolvimento.

Palavras-chave Políticas Públicas, Saúde Sexual e Reprodutiva, Adolescentes e Jovens, Cabo Verde 


\section{Introdução}

Cabo Verde é um arquipélago situado na costa oeste do continente africano composto por dez ilhas, das quais nove habitadas. Apenas $10 \%$ do seu território é de terra arável e o país dispõe de limitados recursos minerais ${ }^{1}$.

Independente desde 1975, nos últimos anos Cabo Verde registrou um rápido crescimento populacional; sua população está estimada de 538.535 habitantes, com uma média de idade de 28 anos; 55\% dos cabo-verdianos residentes se encontra na faixa entre 15 e 49 anos, sendo 20\% entre 15 e 24 anos $^{2}$. Ou seja, há uma importante parcela de pessoas em idade reprodutiva e de adolescentes, jovens.

Após a independência houve um esforço do governo, com apoio da cooperação internacional, de organizar um sistema de saúde capaz de enfrentar as altas taxas de mortalidade por causas evitáveis. Uma das primeiras ações neste sentido foi a criação do programa de Proteção Materno Infantil /Planejamento Familiar e Programa Alargado de Vacinação, PMI/PF/PAV. Em 2001, seguindo a Plataforma de Ação da IV Conferência Internacional sobre População e Desenvolvimento, CIPD, o PMI/PF/PAV foi ampliado visando atingir adolescentes, jovens e homens adultos, passando a chamar-se Programa Nacional de Saúde Reprodutiva, PNSR 3 . Para tanto, foram implantados serviços amigáveis de saúde reprodutiva para adolescentes e jovens, os Centros de Saúde Reprodutiva, CSR; temas de saúde sexual e reprodutiva, SSR foram incorporados às ações educativas realizadas nas comunidades e Centros de Juventude, e tópicos voltados à educação sexual foram incluídos nos currículos escolares ${ }^{3}$

Avaliação do PNSR, realizada em $2007^{4}$, apontou dificuldades de implementação dos CSR em âmbito local, identificando posturas moralistas de profissionais, dúvidas quanto à confidencialidade e receio das meninas de serem vistas frequentando estes serviços, o que denunciaria sua atividade sexual. Estudos posteriores confirmaram a carência de recursos humanos qualificados para a abordagem adequada de questões de SSR com adolescentes e jovens, além de outras lacunas, como a baixa frequência dos homens nos serviços e a sub utilização dos Centros de Juventude ${ }^{5}$.

Aspectos da organização e funcionamento do sistema de saúde do país também foram referidos como entraves ao desempenho do PNSR 6 . O modelo de atenção está centrado no trabalho médico e no hospital, e a implementação de ações de atenção primária e promoção da saúde é di- ficultada pela falta de profissionais qualificados para este tipo de atividade e de integração entre serviços e setores que atuam diretamente com a população.

Características sócio culturais do país foram evidenciadas como obstáculos à implementação do PNSR. Na atual sociedade cabo-verdiana coexistem padrões morais rígidos e desigualdades de gênero herdados do período colonial, e estilos de vida contemporâneos e globalizados ${ }^{7}$. A mescla entre concepções e práticas conservadoras relativas à sexualidade e reprodução, expressões de autonomia das mulheres e comportamentos sexuais liberais permeiam, de forma ambígua e contraditória, a discussão sobre SSR e as ações do PNSR.

Este trabalho busca apontar alcances, limites e desafios do PNSR de Cabo Verde, visando contribuir para o avanço na agenda de SSR do país.

\section{O contexto caboverdiano}

Desde 2008 Cabo Verde é classificado pelo sistema das Nações Unidas como um país de renda média; em 2015 o país figurava em 122 lugar entre 188 países no Îndice de Desenvolvimento Humano (IDH) ${ }^{8}$.

Cabo Verde tem enfrentado desafios ao desenvolvimento e crescimento econômico, relacionados à necessidade de importação de alimentos e escassez de água potável e fontes naturais de ener$\mathrm{gia}^{2}$. A abertura de postos de trabalho não é suficiente para absorver a mão de obra que aumenta com o crescimento populacional, ou para estimular a formação profissional. Em 2011, 49,9\% da população entre 15 e 25 anos não trabalhava nem frequentava a escola9 . Violência urbana, formação de gangues juvenis, comércio sexual e uso de substâncias permeiam a vida dos caboverdianos, ao lado do desemprego e da desocupação ${ }^{10}$.

A distância e diversidade entre as ilhas são desafios para a garantia de acesso da população a bens sociais. Hospitais gerais só estão disponíveis em 02 ilhas e os 06 hospitais regionais, de menor porte, estão localizados nas ilhas de maior densidade populacional. Centros de saúde, CS, postos de saúde e unidades sanitárias básicas estão distribuídos nas ilhas também em função da concentração de habitantes; apenas os CS contam com médicos ou enfermeiros, que periodicamente se deslocam até os postos de saúde para consultas. Havendo necessidade, usuários são levados para as ilhas que oferecem serviços mais complexos. As ações de saúde são prestadas mediante coparticipação: alguns serviços, como o pré natal, orientação contraceptiva e oferta de 
condom são gratuitos; outros, como o exame de papanicolau e aplicação de contraceptivos injetáveis exigem o pagamento de pequenas taxas ${ }^{11}$.

As atividades de SSR são oferecidas nos CS, e visam prioritariamente o acompanhamento pré-natal e oferta de contracepção. Os CSR, num total de 05, estão integrados aos CS. Nas ilhas onde não foram implantados CSR, adolescentes e jovens que precisam de orientação contraceptiva devem buscar os CS. Uma organização não governamental ligada a International Planned $\mathrm{Pa}$ renthood Federation também fornece serviços em SSR de caráter assistencial e educativo, atuando em 05 ilhas ${ }^{11}$.

Segundo o Inquérito Demográfico de Saúde Reprodutiva de $2005^{12}$, a prevalência de uso de contracepção é de 43.9\% entre mulheres unidas. A redução da taxa de fecundidade, de 2.9 filhos por mulher, em 2005, para 2.3, em 2015, sugere expansão desta prática. O declínio da fecundidade é observado em todas as faixas etárias, inclusive entre menores de 19 anos. Contudo, o número de gestações entre jovens ainda é considerado elevado. Em 2013, 20\% das gestantes que realizaram pre-natal tinham 19 anos ou menos ${ }^{4}$; dados de 2010 apontam que $26.2 \%$ das jovens de 15 a 19 anos e $0,5 \%$ com menos de 15 anos já havia engravidado alguma vez na vida ${ }^{12}$.

A legislação permite a interrupção da gravidez em gestações de até 12 semanas e com anuência da família, no caso de menores de 18 anos. Contudo, estima-se que 60\% dos abortos praticados no país ocorram fora das estruturas de saú$\mathrm{de}^{13}$. As razões para o aborto clandestino incluem o não preenchimento dos requisitos legais, como tempo de gestação, aceite da família, pagamento da taxa para o procedimento, ou falta de equipamento de saúde adequado na ilha de residência da gestante. Relatos de maus tratos nos serviços também contribuem para a sua realização de forma clandestina ${ }^{13}$.

A norma que prevê a interrupção dos estudos durante a gravidez é referida como mais um determinante para a prática do aborto, legal ou clandestino. Falta de acesso à contracepção de emergência, seja por produto específico ou pelo método Yuzpe, é mais um fator. Apesar de não haver dados precisos sobre a magnitude do aborto e o perfil das mulheres que o realizam, a sua ocorrência é um dos motivos alegados para a busca de aprimoramento do $\mathrm{PNSR}^{4}$

A prevalência da infecção pelo HIV/aids em Cabo Verde é de $0,8 \%$ na população em geral; $7,9 \%$ entre trabalhadoras do sexo; 3,9\% entre usuários de drogas e 15\% entre homens homossexu- ais. A taxa de detecção entre mulheres é maior do que entre os homens, possivelmente em função da realização de testagem durante o pré natal ${ }^{14}$, mas também em decorrência das barreiras sócio-culturais relativas à homossexualidade, em que pesem evidências de trocas sexuais entre homens ${ }^{14}$. Há pouca integração entre as ações de prevenção e cuidado voltadas para as populações de maior prevalência e as ações voltadas para a população geral. Não há ofertas no CS para profissionais do sexo, usuários de drogas e homens homossexuais e não há fluxos para atender suas demandas em $\mathrm{SSR}^{6}$. O PNSR apenas preve ações de caráter geral visando estimular o uso do preservativo.

Estudos observacionais e com entrevistas face a face, referem resistência ao uso deste insumo $^{10,13}$. Contudo, inqueritos populacionais referem sua utilização por $65 \%$ das pessoas envolvidas em relações ocasionais no último ano ${ }^{14}$. A concepção de que a Aids é uma doença "do outro": migrantes, moradores dos bairros mais pobres e pessoas "promíscuas" também é apontada em alguns estudos ${ }^{5}$. Este tipo de equívoco, ao lado das desigualdades de gênero, traz riscos específicos para as mulheres, que correspondem a 57,3\% dos novos casos de infecção no país ${ }^{14}$.

\section{Alcances e limites do PNSR}

A redução da mortalidade materna e infantil, estimadas em 2011 em 26 e 15 por 100.000 nascidos vivos, respectivamente ${ }^{15}$, é proclamada como uma das principais realizações do $\mathrm{PMI} / \mathrm{PF} / \mathrm{PAV}$. Assim, um dos compromissos do PNSR, desde a sua formulação, foi manter esta conquista, ao lado de buscar novos avanços.

Para tanto, as estratégias do PNSR incluíram a oferta de serviços de SSR- informação e métodos contraceptivos e atenção à saúde para adolescenttes, jovens e homens adultos em unidades de saúde, realização de atividades comunitárias sobre o tema e ações educativas nas escolas. A mudança do nome do programa assinala esta ampliação.

$\mathrm{O}$ exito e pioneirismo do $\mathrm{PMI} / \mathrm{PF} / \mathrm{PAV}$ no país faz com que profissionais e usuários ainda usem a denominação "PMI-PF" para os serviços que realizam ações em SSR. Todavia, a persistência do nome também indica que há poucas ofertas regulares e gratuitas nos CS e CSR além da contracepção e atenção ao ciclo gravídico puerperal, o que restrige o acesso de adolescentes, jovens e homens adultos. A frequência dos CS e CSR continua sendo predominantemente de mulheres grávidas e crianças ${ }^{4-6}$. 
A demanda por consultas reduz a disponibilidade de profissionais para realização de ações educativas que abordam de modo adequado questões de SSR. Há relatos de informações distorcidas e permeadas por juízos de valor e vieses de gênero por parte de técnicos dos CS e CSR, e desconfiança quanto ao seu sigilo, especialmente nas localidades menores, onde profissionais e usuários se conhecem e convivem no $\operatorname{cotidiano}^{4-6}$.

A oferta de materiais de Informação, Educação e Comunicação (IEC) nos CS, CSR, escolas e Centros de Juventude é insuficiente. Assim, o acesso dos seus usuários a informações fica restrito ao que é veiculado pelos profissionais. As famílias, que só após a independência puderam ter contato com serviços de SSR raramente conseguem oferecer suporte a seus filhos nestes assuntos ${ }^{10}$.

As ações de educação sexual nas escolas também apresentam vieses. A abordagem dos temas de SSR em geral é feita no âmbito da disciplina de Formação Pessoal e Social, FPS, por professores que nem sempre têm preparo ou formação e não raro transmitem seus valores e crenças pessoais, deixando os alunos constrangidos para expor suas dúvidas e questões a respeito do tema ${ }^{4-6}$.

As atividades comunitárias voltadas para a SSR são as menos estruturadas, pois dependem da disponibilidade de algum profissional do CS ou do CSR para apresentar e debater os tópicos, e de alguma liderança que organize o evento. Quando ocorrem, apresentam os mesmos problemas das atividades realizadas nas escolas, nos CS e nos CSR: carência de metodologias dialógicas que tornem a atividade mais interessante e participativa, e veiculação de juízos de valor. Discursos a respeito da "irresponsabilidade" dos jovens ou dos efeitos danosos do misoprostol, amplamente utilizado no aborto ilegal, são frequentes nestas oportunidades, denunciando a condenação ao exercício da sexualidade e o reforço das desigualdades de gênero ${ }^{5,6}$.

Assim, embora o PNSR reconheça o direito ao exercício da sexualidade, e admita que a SSR não é um tema apenas de mulheres, mas inclui homens, adolescentes e jovens, a tradução deste reconhecimento em práticas ainda é limitada. $\mathrm{O}$ potencial trazido pelo aumento da cobertura das ações de SSR e das estruturas que oferecem serviços neste tema pode ser mais explorado.

\section{Desafios do PNSR}

Dados os objetivos do PNSR, a discussão sobre a sua efetividade deve incluir a redução da gravidez entre jovens e das gestações não planejadas, o aumento do uso da contracepção, a diminuição do numero de abortos e das novas infecções pelo HIVe ainda maior utilização dos serviços por homens, adolescentes e jovens. Como visto, o alcance destes objetivos esbarra na dinâmica do sistema de saúde e em posturas de profissionais em relação à SSR; assim, os desafios do PNSR dizem respeito a otimização dos recursos disponíveis e criação de consensos que permitam a superação das barreiras culturais identificadas, o que torna relevante repensar o modelo de atenção à saúde vigente.

É necessário compatibilizar os custos e complexidade crescentes do setor com o aumento da demanda e a incorporação tecnológica na saúde e ampliar canais de diálogo com a população, visando a promoção da saúde, incluindo a SSR. Sendo Cabo Verde um país jovem, com uma população jovem e urgência de crescimento econômico, este tema é crucial. Gestações não planejadas e infecção pelo HIV, por exemplo, têm impactos negativos no desenvolvimento ${ }^{16}$. Abre-se, portanto, o desafio de propor um modelo de atenção à saúde que contemple tanto a assistência quanto a promoção de saúde e prevenção de doenças, abordando a SSR na perspectiva da equidade de gênero no contexto da cultura caboverdiana e das subculturas locais ${ }^{17}$. O que exige a construção de ações intersetoriais e mudanças nas concepções de saúde estritamente biomédicas.

Há também que reconsiderar o que pode ser tomado como indicador de sucesso do PNSR. A gravidez entre jovens, por exemplo, não acontece apenas em Cabo Verde ${ }^{18} \mathrm{e}$ não deve ser pensado somente em relação ao acesso a informação e métodos. Este é um evento frequente, especialmente em países pobres e em desenvolvimento, que envolve aspectos culturais e simbólicos além dos sanitários $^{19}$. O Plano Estratégico da Juventude de Cabo Verde, por exemplo, conceitua como jovens as pessoas com a idade entre 15 e 29 anos, embora muitas moças aos 18 anos já se sintam capazes de escolher a maternidade, e rapazes antes dos 29 anos, já se insiram socialmente como adultos ${ }^{20}$. O conjunto de pessoas definidas como adolescentes e jovens no país representa cerca de $25 \%$ da população, e é bastante heterogêneo. Para alguns, as vivências em relação à maternidade, paternidade e sexualidade, podem representar as afirmações possíveis das suas feminilidades, masculinidade e projetos de vida ${ }^{21}$. Ou seja, nem todas as gestações de jovens são um problema para quem as vivencia, embora algumas não sejam planejadas ou desejadas.

Gestações não planejadas ocorrem em vários países, com magnitudes distintas, entre mulheres 
de diferentes idades, e por diferentes razões. Inclui-se aí a percepção de que a gravidez é um evento natural e o planejamento se referiria apenas à decisão de não ter filhos ${ }^{18}$. Assim, a gestação não planejada não se resume a problemas de acesso à contracepção; concepções em torno da reprodução e da maternidade interfrem no seu uso. Neste sentido, a redução das gestações não planejadas em Cabo Verde envolveria tanto o aumento do acesso aos serviços que disponibilizam métodos e do leque de contraceptivos disponíveis, atualmente concentrados nos métodos hormonais, orais ou injetáveis, quanto mudanças culturais no sentido da desnaturalização da maternidade. Considere-se ademais, a importância das oportunidades de autorealização para as mulheres, fundamental para o uso consistente de contraceptivos ${ }^{22}$.

O aborto induzido também é uma prática disseminada no mundo, não se restringindo a mulheres solteiras ou jovens e não derivando apenas da falta de acesso à contracepção. $\mathrm{O}$ reconhecimento de que mesmo quando há acesso a métodos as mulheres engravidam, e decidem não prosseguir a gestação tem fomentado políticas que permitem a interrupção segura da gravidez ${ }^{23}$. Embora em Cabo Verde haja esta possíbilidade, as dificuldades de acesso refletem as controvérsias em torno do tema e tornam-se obstáculos à realização do aborto seguro. $\mathrm{O}$ aprofundamento da discussão sobre o tema e a produção de dados sobre as características desta prática no país é um desafio, que deve ser enfrentado junto com o debate sobre a descriminalização do comércio e uso do misosprostol e disseminação da contracepção de emergência.

Ações educativas com base comunitária voltadas para equidade de gênero podem contribuir para a responsabilização masculina em SSR e redução das gestações e abortos que resultam do sexo forçado ou não consensual ${ }^{24}$; entretanto, a proposta de trazer homens para os serviços de saúde, tal como estes estão organizados talvez não seja estratégia mais adequada para esta finalidade. $\mathrm{O}$ desenho tradicional dos serviços de saúde reprodutiva não atrai a população masculina $^{21}$; seu uso, quando ocorre, fica restrito ao apoio à parceira, não havendo uma apropriação deste espaço pelos homens para a abordagem das questões de SSR de seu interesse.

Cabe também questionar a estratégia de concentrar a prevenção do HIV em grupos específicos. Embora esta seja uma diretriz global, a transição cultural vivida na sociedade caboverdiana torna as redes sexuais especialmente complexas. A focalização da prevenção em grupos específicos acaba por afastar pessoas que têm práticas sexuais de risco, como o sexo sem proteção com pessoas do mesmo sexo ou na troca de sexo por dinheiro, sem necessariamente se identificar com algum dos grupos considerados mais expostos à infecção ${ }^{25}$.

\section{Considerações finais}

Não se pode negar a relevância e oportunidade do PNSR de Cabo Verde. A redução da mortalidade materna e infantil, e ainda a possibilidade de mulheres cabo-verdianas terem controle sobre suas decisões reprodutivas são realizações inegáveis.

As lacunas e desafios do PNSR devem ser considerados tendo em vista o processo de transição sócio cultural por que passa o país, as características do modelo de atenção e a organização das práticas e serviços de saúde, mas também as dificuldades enfrentadas por programas de SSR em países em desenvolvimento e com grande percentual de jovens na sua composição demográfica.

O modelo de atenção vigente, com o profissional médico no centro dos processos de cuidado, apresenta fragilidades e já não corresponde à realidade do país. Há escassez de médicos onde eles são essenciais, e carência de ações de prevenção ou promoção da saúde realizadas por outros profissionais, capacitados para este tipo de atividade e orientados numa perspectiva programática.

Entretanto, a mudança do modelo assistencial, com maior enfase na intersetorialidade, promoção da saúde e fortalecimento das redes de atenção por si só não garante a efetividade do PNSR. Há que ser enfrentado o desafio das diferentes concepções sobre gênero e sexualidade que permeiam as práticas.

A sexualidade, embora seja uma dimensão humana inscrita nos corpos é fundante das subjetividades e, portanto, fluida e dinâmica. Sua abordagem em serviços de saúde organizados para assistência médica, apesar de ser necessária, é insuficiente e muitas vezes reducionista. Daí a exigência de práticas complementares e intersetoriais que possam ter impacto na cultura e nos sentidos socialmente construídos para a sexualidade. Modelos de atenção em saúde com numa atenção primária forte e voltados para a promoção da saúde podem favorecer este diálogo, contribuindo para a criação de novos significados e valores em relação a sexualidade e da saúde sexual.

As propostas do PNSR de Cabo Verde têm um grande potencial de transformação das culturas sexuais locais, seja por meio dos serviços prestados ou pela veiculação de informações, posturas e valores sobre sexo e sexualidade. Suas 
ações devem considerar as dinâmicas sócio-culturais relativas à sexualidade e ao contexto de transição econômica e demográfica por que passa o país, de modo a garantir acesso universal aos direitos sexuais e reprodutivos, integrando a SSR às estratégias e programas nacionais de desenvolvimento, conforme previsto na CIPD e reiterado nos Objetivos de Desenvolvimento Sustentável.

\section{Referências}

1. The World Bank (WB). Country Profile Cape Verde. New York: WB; 2016.

2. Instituto Nacional de Estatística de Cabo Verde (INE). Objetivos de Desenvolvimento do Milenio. Indicadores de Cabo Verde. Praia: INE; 2015

3. Cabo Verde. Ministério da Saúde (MS). Programa Nacional de Saúde Reprodutiva 2008-2012. Praia: MS; 2009.

4. Cabo Verde. Ministério da Saúde (MS). Fundo de População das Nações Unidas (UNFPA). Avaliaçãodo PNSR. Praia: MJEDRH, UNFPA; 2007 Relatório final, mimeo.

5. Cabo Verde. Ministério da Saúde (MS). Fundo de População das Nações Unidas (UNFPA). Avaliação dos serviços de saúde reprodutiva dos adolescentes e jovens em Cabo Verde. Praia: MJEDRH, UNFPA; 2012.

6. Cabo Verde. Ministério da Saúde (MS). Análise da prestação dos cuidados de saúde, incluindo a saúde sexual reprodutiva do homem, da mulher e do adolescente nos centros de saúde de Cabo Verde. Praia: MS; 2015.

7. Anjos JC. Sexualidade juvenil de classes populares em Cabo Verde: os caminhos para a prostituição de jovens urbanas pobres. Revista Estudo Feministas 2005; 13(1):163-177.

8. Programa das Nações Unidas para o Desenvolvimento (PNUD). Relatorio ODM Cabo Verde. Nova Iorque: PNUD; 2015.

9. Instituto Nacional de Estatística de Cabo Verde (INE). IV Recenseamento Geral da População e Habitação 2010. Praia: INE; 2011.

10. Oliveira CS, Lima RW. Estudo sobre abuso e exploração sexual de crianças e adolescentes em Cabo Verde e do plano de aç̧ão para o combate ao abuso e exploração sexual de crianças e adolescentes. Praia: ICCA/Unicef; 2015. Mimeo.

11. Organização Mundial de Saúde (OMS). Governança do Sistema de Saúde da Cabo Verde. Geneva: OMS; 2008. Relatório final, mimeo.

12. Instituto Nacional de Estatística de Cabo Verde (INE). Inquérito Demográfico de Saúde Reprodutiva 2005. Praia: INE; 2008.

13. Afrosondagem. Estudo sobre o aborto em Cabo Verde. Praia: VERDEFAM, ICIEG; 2012.

14. Comitê da Coordenação do Combate à SIDA (CCS-SIDA). Rapport de Progrès sur la riposte au SIDA au Cabo Verde. Paris: UNAIDS; 2015.

15. Ministério da Saúde. Relatório Estatístico de Saúde, 2013. Praia, 2014, mimeo

\section{Colaboradores}

WV Villela, RW Lima e CF Brito participaram igualmente do levantamento de dados empíricos e bibliográficos que deram origem a estas reflexões, sua análise e discussão e da redação do manuscrito.
16. Germain A, Sen G, Garcia-Moreno C, Shankar M. Advancing sexual and reproductive health and rights in low- and middle-income countries: Implications for the post-2015 global development agenda, Global Public Health 2015; 10(2):137-148.

17. Albuquerque TIP, Pereira RM, Araújo Júnior JLAC. Perspectivas e desafios da "nova" Política Nacional de Promoção da Saúde: para qual arena política aponta a gestão? Cien Saude Colet 2016; 21(6):1695-1706.

18. Kirkman M, Stubber C, Rowe H, Holton S, Bayly C, Jordan L, Fisher J. Subjective meanings of 'unintended' pregnancy: interviews from understanding fertility management in contemporary Australia. Cult Health Sex 2016; 19(2):179-193.

19. Sedgh G, Finer LB, Bankole A, Eilers MA, Singh S. Adolescent Pregnancy, Birth, and Abortion Rates Across Countries: Levels and Recent Trends. J Adolesc Health 2015; 56(2):223-230.

20. Cabo Verde. Ministério da Juventude, Educação e Direitos Humanos (MJEDRH). Plano Estratégico da Juventude. Praia: MJEDRH; 2013.

21. Onyango M, Owoko S, Oguttu M. Factors that Influence Male Involvement in Sexual and Reproductive Health in Western Kenya: A Qualitative Study. Afr J Reprod Health 2010; 14(4):32-42.

22. Wellings $\mathrm{K}$, Jones KG, Mercer CH, Tanton C, Clifton S, Datta J, Johnson AM. The prevalence of unplanned pregnancy and associated factors in Britain: findings from the third National Survey of Sexual Attitudes and Lifestyles. Natsal-3. Lancet 2013; 382(9907):1807-1816.

23. Guttmacher Institute. Induced Abortion worldwide. Global incidence and trends. Fact sheet, May, 2016. [acessado 2016 Nov 26]. Disponível em: https://www.guttmacher.org/fact-sheet/induced-abortion-worldwide

24. Heidari S, García Moreno C. Gender-based violence: a barrier to sexual and reproductive health and rights. Reproductive Health Matters 2016; 24(47):1-4.

25. Greco D. Trinta anos de enfrentamento à epidemia da Aids no Brasil, 1985-2015. Cien Saude Colet 2016; 21(5):1553-1564.

Artigo apresentado em 19/09/2016

Aprovado em 02/02/2017

Versão final apresentada em 04/02/2017 